\title{
Genetic association analysis of 13 nuclear-encoded mitochondrial candidate genes with type II diabetes mellitus: the DAMAGE study
}

Erwin Reiling ${ }^{1}$, Jana V van Vliet-Ostaptchouk ${ }^{2}$, Esther van 't Riet ${ }^{3}$, Timon W van Haeften ${ }^{4}$, Pascal A Arp ${ }^{5}$, Torben Hansen ${ }^{6}$, Dennis Kremer ${ }^{7}$, Marlous J Groenewoud ${ }^{1}$, Els C van Hove ${ }^{1}$, Johannes A Romijn ${ }^{8}$, Jan WA Smit ${ }^{8}$, Giel Nijpels ${ }^{3}$, Robert J Heine ${ }^{3}$, André G Uitterlinden ${ }^{5}$, Oluf Pedersen $^{6,9,10}$, P Eline Slagboom ${ }^{7}$, Johannes A Maassen ${ }^{1,3}$, Marten H Hofker ${ }^{2}$, Leen $\mathrm{M}$ 't Hart*,1,11 and Jacqueline M Dekker',11

\footnotetext{
${ }^{1}$ Department of Molecular Cell Biology, Leiden University Medical Center, Leiden, The Netherlands; ${ }^{2}$ Department of Pathology and Laboratory Medicine, University Medical Center Groningen, Groningen, The Netherlands; ${ }^{3}$ VU Medical Center, EMGO institute, Amsterdam, The Netherlands; ${ }^{4}$ Department of Internal Medicine, University Medical Center Utrecht, Utrecht, The Netherlands; ${ }^{5}$ Department of Internal Medicine, Erasmus University Medical Center, Rotterdam, The Netherlands; ${ }^{6}$ Steno Diabetes Center and Hagedorn Research Institute, Gentofte, Denmark; ${ }^{7}$ Department of Medical Statistics, Leiden University Medical Center, Leiden, The Netherlands; ${ }^{8}$ Department of Endocrinology, Leiden University Medical Center, Leiden, The Netherlands; ${ }^{9}$ Faculty of Health Science, Aarhus University, Aarhus, Denmark;

${ }^{10}$ Faculty of Health Science, University of Copenhagen, Copenhagen, Denmark
}

Mitochondria play an important role in many processes, like glucose metabolism, fatty acid oxidation and ATP synthesis. In this study, we aimed to identify association of common polymorphisms in nuclear-encoded genes involved in mitochondrial protein synthesis and biogenesis with type II diabetes mellitus (T2DM) using a two-stage design. In the first stage, we analyzed 62 tagging single nucleotide polymorphisms (SNPs) in the Hoorn study ( $n=999$ participants) covering all common variation in 13 biological candidate genes. These 13 candidate genes were selected from four clusters regarded essential for correct mitochondrial protein synthesis and biogenesis: aminoacyl tRNA synthetases, translation initiation factors, tRNA modifying enzymes and mitochondrial DNA transcription and replication. SNPs showing evidence for association with T2DM were measured in second stage genotyping ( $n=10164$ participants). After a meta-analysis, only one SNP in SIRT4 (rs2522138) remained significant $(P=0.01)$. Extending the second stage with samples from the Danish Steno Study ( $n=1220$ participants) resulted in a common odds ratio (OR) of $0.92(0.85-1.00)$, $P=0.06$. Moreover, in a large meta-analysis of three genome-wide association studies, this SNP was also not associated with T2DM $(P=0.72)$. In conclusion, we did not find evidence for association of common variants in 13 nuclear-encoded mitochondrial proteins with T2DM.

European Journal of Human Genetics (2009) 17, 1056-1062; doi:10.1038/ejhg.2009.4; published online 11 February 2009

Keywords: type II diabetes mellitus; candidate gene; SNP; mitochondria

*Correspondence: Dr LM 't Hart, Department of Molecular Cell Biology Leiden University Medical Center (LUMC), PO Box 9600, 2300RC Leiden, The Netherlands. Tel: + 31715269266; Fax: + 31715268270;

E-mail: I.m.t_hart@lumc.nl

${ }^{11}$ These authors have contributed equally to this work.

Received 22 July 2008; revised 9 January 2009; accepted 14 January 2009; published online 11 February 2009

\section{Introduction}

Mutations in genes involved in mitochondrial protein synthesis have been found to associate with a diabetic state. The $3243 \mathrm{~A}>\mathrm{G}$ mutation in the mitochondrial tRNA 
(Leu, UUR) gene, which impairs mitochondrial protein synthesis, is strongly diabetogenic, as are some additional mutations in mtDNA-encoded tRNA genes. ${ }^{1,2}$ Recently, we found that an H324Q variant in the nuclear-encoded mitochondrial leucyl tRNA synthetase (LARS2) results in an increased type II diabetes mellitus (T2DM) susceptibility. ${ }^{3}$ It seems that a change in the activity of mitochondrial protein synthesis makes the organism more vulnerable to develop glucose intolerance.

A change in mitochondrial protein synthesis is expected to result in an unbalance in the stoichiometry of the proteins composing the respiratory chain. Some proteins of the respiratory chain are synthesized through the mitochondrial protein synthesis machinery, whereas the others are synthesized in the cytosol and imported into the mitochondrion. Therefore, impaired activity of the mitochondrial protein synthesis machinery is expected to result in an unbalance between the nuclear DNA- and mitochondrial DNA-encoded proteins of the respiratory chain. A similar situation of an unbalance may arise when the rate of mitochondrial biogenesis is altered.

In T2DM, a reduced activity of the respiratory chain is seen, which is also present in the first degree relatives. ${ }^{4,5}$ The biochemical basis of this phenomenon may arise through an unbalanced expression of the various proteins. On the basis of these considerations, we hypothesized that polymorphisms in genes involved in mitochondrial protein synthesis and mitochondrial biogenesis may alter the balanced expression of proteins of the respiratory chain. As a result, these polymorphisms are expected to modify the risk for T2DM. It is estimated that the complete mitochondrial proteome consists of approximately 1500 proteins, which are mostly encoded by the nuclear genome. ${ }^{6}$ Common variations in genes encoded by the mitochondrial genome are not associated with the onset of T2DM. ${ }^{7,8}$ Based on a literature study, we selected 13 candidate genes divided in several clusters. These candidates are all nuclear genes encoding for mitochondrial proteins.

Owing to the previous association of LARS2 with T2DM, the first cluster consisted of the mitochondrial aminoacyltRNA synthetases DARS2, TARS2 and IARS2. DARS2 and TARS2 are located on a widely replicated T2DM linkage locus on chromosome 1q. ${ }^{9}$ Furthermore, variants in the tRNA $^{\text {Ile }}$ gene were shown to be associated with metabolic disorders. ${ }^{10}$

The second cluster consisted of the two mitochondrial translation initiation factors, MTIF2 and MTIF3. These two genes are involved in initiation of the mitochondrial protein synthesis and their dysfunction could lead to altered expression of mitochondrial proteins, and subsequently to oxidative stress and late-onset disease. ${ }^{11-13} \mathrm{We}$ have selected them above other important translation factors, like TUFM, TSFM and GFM1, because mutations in the latter have already been associated with severe early-onset disease. ${ }^{14}$
The third cluster consisted of MTO1, TRMU and GTPBP3, which encode tRNA modifying enzymes. Variation in these genes could lead to dysfunctional enzymes and subsequently result in impaired tRNA modification and mitochondrial disease. ${ }^{15-17}$

In our fourth cluster, we included TFAM and PPRC1, which are involved in transcription and replication of the mitochondrial genome. It has been shown earlier that a low mitochondrial copy number is associated with T2DM, although this is not confirmed in all studies. ${ }^{18,19}$ TFAM is one of the main regulators of mitochondrial transcription and replication, and thus of the mitochondrial copy number. $^{20,21}$ PPRC1 is a PPARGC1A-related gene and involved in mitochondrial biogenesis. ${ }^{22}$

In addition, we selected three genes, which did not fit in a specific cluster but may affect mitochondrial biogenesis and function. SIRT3 and SIRT4, which are Sir2 homologs, are involved in mitochondrial function and insulin secretion. $^{23,24}$ Moreover, SIRT3 has been associated with longevity. ${ }^{25}$ GPAM is the final candidate gene, which is involved in triacylglycerol and phospholipid synthesis. GPAM knockout mice show hyperinsulinaemia and reduced glucose tolerance. ${ }^{26,27}$ Therefore, we hypothesized that defects in GPAM could lead to insulin resistance and subsequently to T2DM.

Dysfunction of these 13 candidate genes potentially affects mitochondrial function and may therefore increase T2DM susceptibility. Therefore, the aim of this study was to analyze the association of common variants in these gene loci with T2DM, using a tagging single nucleotide polymorphism (SNP) approach.

\section{Materials and methods Single nucleotide polymorphism selection}

Tagging SNPs were selected using the HapMap database (www. HAPMAP.org) and Tagger. ${ }^{28,29}$ The threshold for the minor allele frequency (MAF) was set at 0.05. Using Tagger, 62 SNPs were selected, which cover all common variation in the selected genes according to HapMap data (phase 2, April 2007 (Build 36), population CEU, $r^{2}>0.8$ ). Aggressive tagging with two- and three-marker tests was used. This resulted in four multimarker tests. The remaining tests were all single marker tests. Gene boundaries include $5^{\prime}$ and $3^{\prime}$-UTR regions. Details about the SNP selection for the individual genes, including gene boundaries, are summarized in Table 1.

\section{Genotyping and quality control}

A two-stage design was used. For first-stage genotyping, the Sequenom platform (Sequenom, San Diego, CA, USA) was used. First, the allelic discrimination plots were visually observed for good clustering. Assays with bad clustering were not further analyzed. SNPs with a success rate below 95\% or not obeying Hardy-Weinberg Equilibrium 
Table 1 Gene boundaries and number of SNPs in the selected candidate genes

\begin{tabular}{|c|c|c|c|c|}
\hline Gene & Chromosome & $\begin{array}{l}\text { Gene boundaries } \\
\text { for SNP selection }\end{array}$ & $\begin{array}{l}\text { Number of SNPs } \\
\text { (successful) }\end{array}$ & $\begin{array}{c}\text { \% covered } \\
(M A F>0.05)\end{array}$ \\
\hline TARS2 & 1 & $148726544-148746371$ & $4(4)$ & 100 \\
\hline DARS2 & 1 & $172060581-172094305$ & $2(1)$ & 28 \\
\hline IARS2 & 1 & $218334078-218387999$ & $6(4)$ & 81 \\
\hline MTIF2 & 2 & $55317262-55349888$ & $7(7)$ & 100 \\
\hline MTO1 & 6 & $74228209-74267896$ & $5(5)$ & 100 \\
\hline TFAM & 10 & $59815181-59825901$ & $5(5)$ & 100 \\
\hline PPRC1 & 10 & $103882777-103900078$ & $3(3)$ & 100 \\
\hline GPAM & 10 & $113899611-113933507$ & $6(6)$ & 100 \\
\hline SIRT3 & 11 & $205031-226361$ & $10(10)$ & 100 \\
\hline SIRT4 & 12 & $119224546-119235427$ & $3(3)$ & 100 \\
\hline MTIF3 & 13 & $26907783-26922711$ & $3(3)$ & 100 \\
\hline GTPBP3 & 19 & $17309379-17314530$ & $4(4)$ & 100 \\
\hline TRMU & 22 & $45109962-45131900$ & $4(3)$ & 81 \\
\hline
\end{tabular}

Gene boundaries and coverage according to HapMap data. Gene boundaries according to genome build 36 .

The widest gene boundaries were used when more isoforms were known.

Table 2 Descriptive statistics of the first and second stage

\begin{tabular}{lcccc}
\hline & & First stage & & Second stage \\
& Controls & Cases & Controls & Cases \\
\hline Participants (\% male) & $519(55)$ & $480(52)$ & $7620(44)$ & $2544(45)$ \\
Age - years (SD) & $65(8)$ & $67(8)$ & $63(12)$ & $68(12)$ \\
BMl - kg/m 2 (SD) & $26(5)$ & $29(5)$ & $26(4)$ & $27(5)$ \\
FPG - mmol/I (SD) & $5.4(0.4)$ & $7.7(2.2)$ & $5.5(0.5)^{\mathrm{a}}$ & $8.3(2.5)^{\mathrm{a}}$ \\
\hline
\end{tabular}

FPG, fasting plasma glucose.

First stage consists of participants from the Hoorn study. ${ }^{30}$ Second stage is a pooled sample of participants originally from the NHS, Breda and ERGO studies. ${ }^{33-35}$

${ }^{a}$ Available for 2438 controls and 334 cases.

$(P<0.01)$ were excluded from analysis. SNPs, which did not fit in the Sequenom assay or failed quality control (QC), were genotyped with Taqman SNP genotyping assays (Applied Biosystems, Foster City, CA, USA) and evaluated with the same QC guidelines. Duplicate samples ( 5\%) showed identical genotypes. SNPs showing evidence for association with T2DM $(P<0.05)$ were selected for secondstage genotyping. Taqman SNP genotyping assays were used for this.

\section{Study cohorts}

The first-stage genotyping was performed in the Hoorn study. ${ }^{30}$ This population-based study consisted of 519 normal glucose tolerant (NGT) participants (aged $65 \pm 8$ years, 55\% male) and 480 T2DM participants (aged $67 \pm 8$ years, 52\% male). Glucose tolerance was tested using a fasting oral glucose tolerance test (OGTT), according to World Health Organization (WHO) criteria. ${ }^{31}$

For the second-stage genotyping, three cohorts from the Netherlands were pooled. The first cohort was the New Hoorn Study (NHS), which is an ongoing, second non-overlapping population-based study in Hoorn. ${ }^{32}$ From this study, we randomly selected 1517 NGT and 147 T2DM participants. All participants underwent an OGTT according to WHO criteria. $^{31}$ To increase power, we included 674 participants with T2DM from the diabetes clinics of the Leiden University Medical Center (LUMC, Leiden, The Netherlands) and from the Vrije Universiteit medical center (VUmc, Amsterdam, The Netherlands). All participants were Dutch caucasians. In total, the NHS sample included 1517 controls (aged $53 \pm 7$ years, 44\% male) and 821 cases (aged $61 \pm 11$ years, 50\% male).

The second cohort was the Breda study. ${ }^{33,34}$ This casecontrol study consisted of 920 healthy controls (according to self report) from the Dutch blood bank (aged $48 \pm 13$ years, 61\% male). 501 cases (aged $71 \pm 10$ years, $46 \%$ male) were $\mathrm{T} 2 \mathrm{DM}$ patients according to WHO criteria. ${ }^{31}$

The third cohort was the ERGO study from Rotterdam. ${ }^{35}$ This population-based study consisted of 5183 NGT participants (aged $69 \pm 9$ years, 41\% male) and 1222 (73 \pm 9 years, $39 \%$ male) T2DM patients.

In total, for the second-stage genotyping, we used 7620 controls and 2544 T2DM cases. The characteristics of both stages are summarized in Table 2 (Characteristics of the independent cohorts are summarized in Supplementary Table S1.). For additional replication of our strongest signal, we extended the second stage with samples from the Danish Steno study. ${ }^{36}$ This case-control study 
consisted of 514 NGT controls (aged $57 \pm 10,46 \%$ male) randomly selected from public registers at the Steno Diabetes Center and the Research Center for Prevention and Health. The 706 cases (aged $59 \pm 10$ years, $48 \%$ male) were recruited from the Steno Diabetes Center.

\section{Statistical analysis}

Differences in genotype distribution and allele frequency were analyzed using a $\chi^{2}$ test. An additive model was the best fit for most of the SNPs. rs1049432 (TFAM) and rs4917960 (PPRC1) might better fit a recessive model, and were therefore also analyzed with this model. A common allelic odds ratio (OR) covering both stages was calculated in a meta-analysis using a Mantel-Haenszel test. Homogeneity of ORs between the different cohorts was tested using a Tarone's test. Results from OGTT (only NGT participants) were analyzed with univariate analysis of variance, using additive, dominant and recessive models, and correction for age, BMI and gender, as possible confounders. Power calculations were performed using Quanto. ${ }^{37}$ Power to detect modest effects in the first stage was limited $(80 \%$ power to detect, OR $1.45, \mathrm{MAF}=0.1)$. Therefore, we did not correct for multiple testing but rather performed extensive replication of all signals from the first stage with $P<0.05$. All statistics were calculated using SPSS 14.0 (SPSS Inc., Chicago, IL, USA).

\section{Results}

Of the 62 SNPs tested in the first-stage genotyping, 58 SNPs passed QC guidelines. Of these SNPs, we selected the top seven SNPs for second-stage genotyping $(P<0.05)$. Data from genome-wide association studies (GWAS), which became available after completion of our study, resulted in the inclusion of two additional SNPs, rs4397793 in TFAM and rs2792751 in GPAM. Compared with our data, both showed a similar effect in at least one of the publicly available databases of GWAS. ${ }^{38,39}$ In total, nine SNPs were selected for genotyping in the second stage (Table 3 ).
We analyzed the second-stage results for rs1049432 in TFAM and rs4917960 in PPRC1 first with a recessive model, because that was the best fit for the first-stage data $\left(P_{\text {rec }}=0.002\right.$ and $P_{\text {rec }}=0.07$, respectively). However, second-stage results showed no support for this model; therefore, the additive model was used for further analyses. There was no evidence of heterogeneity of ORs between the independent cohorts; therefore, we pooled all the second-stage data. A meta-analysis of both stages was performed to calculate a common OR for the nine selected SNPs (Table 3). Only the G allele of rs2522138 in SIRT4 remained significant $(P=0.01)$, but this was mainly caused by first-stage results. To confirm the observed association, we expanded the second stage with the Danish Steno study resulting in a common OR of 0.92 (0.85-1.00), $P=0.06$.

The C allele of rs4917960 in PPRC1 was borderline significant after the meta-analysis $(\mathrm{OR}=1.06(1.00-1.13)$ $P=0.06)$. This association was nominal and mainly caused by first-stage data. The ORs in all independent cohorts are shown in Supplementary Table S2. Correction for age, gender and BMI (when available) did not influence the results.

Finally, we analyzed OGTT data for association of the selected genes with differences in clinical variables like glucose and insulin. Furthermore, association with BMI was assessed when the data were available. No differences were observed using different models (data not shown).

After completion of our study, a meta-analysis of three T2DM GWAS appeared. ${ }^{40}$ The coverage of common variation in the 13 selected genes in the GWAS was between 80 and $100 \%$ except GTPBP3, which was covered for $50 \%$. We compared our results with the GWAS data, using the OR-based analysis (Table 4). The G allele of rs2522138 in SIRT4 was not associated with T2DM susceptibility in the GWAS data $(\mathrm{OR}=1.02(0.93-1.11), P=0.72)$. The $\mathrm{C}$ allele of rs4917960 in PPRC1 showed an OR of 1.04 (0.97-1.12), $(P=0.23)$ in the GWAS data, which is in the same direction as observed in our study $(\mathrm{OR}=1.06(1.00-1.13), P=0.06)$. Two SNPs located in IARS2 (the C alleles of rs17007135 and

Table 3 Results of first and second-stage genotyping

\begin{tabular}{|c|c|c|c|c|c|c|c|c|c|}
\hline \multirow[b]{2}{*}{ rs number } & \multirow[b]{2}{*}{ Gene } & \multicolumn{2}{|c|}{$M A F^{\mathrm{a}}$} & \multicolumn{2}{|l|}{ First Stage } & \multicolumn{2}{|c|}{ Second Stage } & \multicolumn{2}{|c|}{ Meta-analysis } \\
\hline & & Controls & Cases & OR $(95 \% \mathrm{Cl})$ & $\mathrm{P}_{\text {Add }}$ & OR $(95 \% \mathrm{Cl})$ & $\mathrm{P}_{\text {Add }}$ & OR $(95 \% \mathrm{Cl})$ & $\mathrm{P}_{\text {Add }}$ \\
\hline rs1937 & TFAM & 0.14 & 0.09 & $0.66(0.50-0.88)$ & 0.005 & $1.00(0.86-1.17)$ & 0.97 & $0.91(0.79-1.04)$ & 0.17 \\
\hline rs4397793 & TFAM & & 0.3 & $0.86(0.72-1.03)$ & 0.10 & $1.04(0.97-1.11)$ & 0.25 & $1.02(0.95-1.08)$ & 0.62 \\
\hline rs1049432 & TFAM & 0.19 & 0.14 & $0.77(0.61-0.97)$ & 0.03 & $1.00(0.91-1.09)$ & 0.95 & $0.97(0.89-1.05)$ & 0.39 \\
\hline rs4917960 & PPRC1 & 0.38 & 0.42 & $1.12(1.00-1.43)$ & 0.05 & $1.05(0.98-1.12)$ & 0.21 & $1.06(1.00-1.13)$ & 0.06 \\
\hline rs17129583 & GPAM & 0.12 & 0.16 & $1.32(1.02-1.70)$ & 0.04 & $0.92(0.84-1.02)$ & 0.12 & $0.97(0.88-1.06)$ & 0.50 \\
\hline rs2792751 & GPAM & 0.26 & 0.24 & $0.87(0.71-1.06)$ & 0.18 & $1.01(0.94-1.09)$ & 0.81 & $0.99(0.93-1.06)$ & 0.82 \\
\hline rs535716 & SIRT3 & 0.24 & 0.19 & $0.78(0.63-0.96)$ & 0.02 & $1.05(0.97-1.13)$ & 0.25 & $1.01(0.94-1.09)$ & 0.78 \\
\hline rs2522138c & SIRT4 & 0.18 & 0.13 & $0.69(0.54-0.88)$ & 0.003 & $0.93(0.85-1.02)$ & 0.13 & $0.92(0.85-1.00)$ & 0.06 \\
\hline rs17085633 & MTIF3 & 0.44 & 0.50 & $1.25(1.05-1.50)$ & 0.01 & $1.02(0.97-1.09)$ & 0.55 & $1.05(0.98-1.11)$ & 0.15 \\
\hline
\end{tabular}

${ }^{\mathrm{a}} \mathrm{MAF}$ in first-stage genotyping (Hoorn study).

${ }^{b}$ Second-stage genotyping only in NHS and Breda study $(n=3759)$.

${ }^{c}$ Meta-analysis includes Steno study. 
Table 4 Comparison of the results of the T2DM GWAS meta-analysis and our replication SNPS

\begin{tabular}{|c|c|c|c|c|c|}
\hline \multirow[b]{2}{*}{ rs number } & \multirow[b]{2}{*}{ Gene } & \multicolumn{2}{|c|}{ Meta-analysis GWAS } & \multicolumn{2}{|c|}{ Meta-analysis of our study } \\
\hline & & OR $(95 \% \mathrm{Cl})$ & P-value & OR $(95 \% \mathrm{Cl})$ & P-value \\
\hline rs1937 & TFAM & $1.02(0.91-1.14)$ & 0.76 & $0.91(0.79-1.04)$ & 0.17 \\
\hline rs4397793 & TFAM & $1.03(0.96-1.09)$ & 0.43 & $1.02(0.95-1.08)$ & 0.62 \\
\hline rs1049432 & TFAM & $1.02(0.94-1.12)$ & 0.69 & $0.97(0.89-1.05)$ & 0.39 \\
\hline rs4917960 & PPRC1 & $1.04(0.97-1.12)$ & 0.23 & $1.06(1.00-1.13)$ & 0.06 \\
\hline rs17129583 & GPAM & $1.00(0.92-1.09)$ & 0.99 & $0.97(0.88-1.06)$ & 0.50 \\
\hline rs2792751 & GPAM & $1.04(0.98-1.11)$ & 0.22 & $0.99(0.93-1.06)$ & 0.82 \\
\hline rs535716 & SIRT3 & $1.03(0.96-1.11)$ & 0.42 & $1.01(0.94-1.09)$ & 0.78 \\
\hline rs2522138 & SIRT4 & $1.02(0.93-1.11)$ & 0.72 & $0.92(0.85-1.00)$ & 0.06 \\
\hline rs17085633 & MTIF3 & $1.00(0.94-1.06)$ & 0.89 & $1.05(0.98-1.11)$ & 0.15 \\
\hline
\end{tabular}

Meta-analysis of three GWAS from the DIAGRAM consortium, $n=10128$ (4549 cases and 5579 controls). ${ }^{40}$ ORs are fixed-effect results.

rs2289191) showed evidence of nominal association in the GWAS meta-analysis $\left(P=6 \times 10^{-4}\right.$ and $P=0.003$, respectively). Unfortunately, the assays for these two SNPs failed in our study. None of the other SNPs in our 13 genes showed evidence of association in the T2DM GWAS metaanalysis.

\section{Discussion}

We analyzed 58 tagging SNPs in 13 genes involved in mitochondrial function for association with T2DM susceptibility using a two-stage design. After meta-analysis of the data of both stages, only rs2522138 in SIRT4 remained borderline significant. However, after extension of the second stage with samples from the Steno study, this association did not remain significant.

For several gene variants measured in our study, there is substantial heterogeneity of ORs between the first and second stage, but not within each stage (Table 3 and Supplementary Table S2). Heterogeneity between the independent cohorts might be caused by the differences in the age, gender or selection criteria used, and might obscure true association (Supplementary Table S1). However, we were unable to identify the reasons for this heterogeneity in our study. Differences in LD between the measured and causal SNP are also an unlikely cause, because the study participants in our cohorts are from similar ancestry. ${ }^{41}$ In the context of testing multiple SNPs, our nominal significant results in SIRT4 and PPRC1 should be interpreted as consistent with statistical noise.

One of the limitations of our study is that it is statistically underpowered to detect SNPs with a very small impact or low allele frequency. Although power in the first stage alone was low, we had at least a power of $80 \%$ at an observed $\mathrm{MAF} \geqslant 0.13$ to detect ORs comparable to those recently reported in GWAS (1.12-1.37) when first and second stage are combined. ${ }^{40}$ Our negative results for these 13 genes are confirmed by the DIAGRAM GWAS metaanalysis, which has a much higher power to detect also more modest effects. ${ }^{38-40,42}$ Taken together, we can exclude that common variants in the selected genes have a major impact on T2DM susceptibility. Rare variants are not covered by our approach; therefore, we cannot exclude a role for such variants. Deep sequencing and analysis of novel rare variants in large well-phenotyped cohorts should provide more insight into these important issues.

Other nuclear-encoded mitochondrial genes are also not among the top hits of reported T2DM GWAS. ${ }^{38-40,42,43}$ Nevertheless, earlier studies suggested that they may be involved in T2DM susceptibility. ${ }^{3,44-47}$ Further combined and detailed analysis of the available GWAS datasets, and analysis of copy number and so-called low-frequency intermediate-penetrance variants are necessary to fully explore the role of nuclear-encoded mitochondrial genes in the pathogenesis of T2DM.

In conclusion, we were unable to detect association of common SNPs in 13 nuclear-encoded mitochondrial candidate genes with T2DM or with related continuous traits.

\section{Acknowledgements}

This study was supported by the Netherlands Organization for Scientific Research, ZonMW RIDE program and the Dutch Diabetes Research Foundation. The Danish study was supported by grants from the Lundbeck Foundation Centre of Applied Medical Genomics for Personalized Disease Prediction, Prevention and Care (LUCAMP), the Danish Health Research Council, and The European Union (EUGENE2, Grant no. LSHM-CT-2004-512013).

The authors would like to acknowledge the participants of all study cohorts for their cooperation.

\section{References}

1 van den Ouweland JM, Lemkes HH, Ruitenbeek W et al: Mutation in mitochondrial tRNA(Leu)(UUR) gene in a large pedigree with maternally transmitted type II diabetes mellitus and deafness. Nat Genet 1992; 1: 368-371.

2 Maassen JA, t Hart LM, Janssen GM, Reiling E, Romijn JA, Lemkes $\mathrm{HH}$ : Mitochondrial diabetes and its lessons for common Type 2 diabetes. Biochem Soc Trans 2006; 34 (Pt 5): 819-823. 
3 't Hart LM, Hansen T, Rietveld I et al: Evidence that the mitochondrial leucyl tRNA synthetase (LARS2) gene represents a novel type 2 diabetes susceptibility gene. Diabetes 2005; 54: $1892-1895$.

4 Kelley DE, He J, Menshikova EV, Ritov VB: Dysfunction of mitochondria in human skeletal muscle in type 2 diabetes. Diabetes 2002; 51: 2944-2950.

5 Mootha VK, Lindgren CM, Eriksson KF et al: PGC-1alpharesponsive genes involved in oxidative phosphorylation are coordinately downregulated in human diabetes. Nat Genet 2003; 34: $267-273$

6 Calvo S, Jain M, Xie X et al: Systematic identification of human mitochondrial disease genes through integrative genomics. Nat Genet 2006; 38: 576-582.

7 Mohlke KL, Jackson AU, Scott LJ et al: Mitochondrial polymorphisms and susceptibility to type 2 diabetes-related traits in Finns. Hum Genet 2005; 118: 245-254.

8 Saxena R, de Bakker PI, Singer K et al: Comprehensive association testing of common mitochondrial DNA variation in metabolic disease. Am J Hum Genet 2006; 79: 54-61.

9 McCarthy MI: Growing evidence for diabetes susceptibility genes from genome scan data. Curr Diab Rep 2003; 3: $159-167$.

10 Wilson FH, Hariri A, Farhi A et al: A cluster of metabolic defects caused by mutation in a mitochondrial tRNA. Science 2004; 306 : $1190-1194$

11 Liao HX, Spremulli LL: Identification and initial characterization of translational initiation factor 2 from bovine mitochondria. J Biol Chem 1990; 265: 13618-13622.

12 Koc EC, Spremulli LL: Identification of mammalian mitochondrial translational initiation factor 3 and examination of its role in initiation complex formation with natural mRNAs. J Biol Chem 2002; 277: 35541-35549.

13 Abahuni N, Gispert S, Bauer $\mathrm{P}$ et al: Mitochondrial translation initiation factor 3 gene polymorphism associated with Parkinson's disease. Neurosci Lett 2007; 414: $126-129$.

14 Scheper GC, van der Knaap MS, Proud CG: Translation matters: protein synthesis defects in inherited disease. Nat Rev Genet 2007; 8: $711-723$.

$15 \mathrm{Li}$ R, Li X, Yan Q, Qin MJ, Guan MX: Identification and characterization of mouse MTO1 gene related to mitochondrial tRNA modification. Biochim Biophys Acta 2003; 1629 (1-3): $53-59$.

16 Umeda N, Suzuki T, Yukawa $M$ et al: Mitochondria-specific RNA-modifying enzymes responsible for the biosynthesis of the wobble base in mitochondrial tRNAs. Implications for the molecular pathogenesis of human mitochondrial diseases. J Biol Chem 2005; 280: 1613-1624.

17 Li X, Guan MX: A human mitochondrial GTP binding protein related to tRNA modification may modulate phenotypic expression of the deafness-associated mitochondrial 12S rRNA mutation. Mol Cell Biol 2002; 22: 7701-7711.

18 Song J, Oh JY, Sung YA, Pak YK, Park KS, Lee HK: Peripheral blood mitochondrial DNA content is related to insulin sensitivity in offspring of type 2 diabetic patients. Diabetes Care 2001; 24: $865-869$.

19 Singh R, Hattersley AT, Harries LW: Reduced peripheral blood mitochondrial DNA content is not a risk factor for Type 2 diabetes. Diabet Med 2007; 24: 784-787.

20 Parisi MA, Clayton DA: Similarity of human mitochondrial transcription factor 1 to high mobility group proteins. Science 1991; 252: 965-969.

21 Ekstrand MI, Falkenberg M, Rantanen A et al: Mitochondrial transcription factor A regulates mtDNA copy number in mammals. Hum Mol Genet 2004; 13: 935-944.

22 Andersson U, Scarpulla RC: Pgc-1-related coactivator, a novel, serum-inducible coactivator of nuclear respiratory factor 1-dependent transcription in mammalian cells. Mol Cell Biol 2001; 21: 3738-3749.
23 Shi T, Wang F, Stieren E, Tong Q: SIRT3, a mitochondrial sirtuin deacetylase, regulates mitochondrial function and thermogenesis in brown adipocytes. J Biol Chem 2005; 280: 13560-13567.

24 Argmann C, Auwerx J: Insulin secretion: SIRT4 gets in on the act. Cell 2006; 126: 837-839.

25 Yamamoto H, Schoonjans K, Auwerx J: Sirtuin functions in health and disease. Mol Endocrinol 2007; 21: 1745-1755.

26 Bell RM, Coleman RA: Enzymes of glycerolipid synthesis in eukaryotes. Annu Rev Biochem 1980; 49: 459-487.

27 Hammond LE, Neschen S, Romanelli AJ et al: Mitochondrial glycerol-3-phosphate acyltransferase-1 is essential in liver for the metabolism of excess acyl-CoAs. I Biol Chem 2005; 280: 25629-25636.

28 The International HapMap Consortium: A haplotype map of the human genome. Nature 2005; 437: 1299-1320.

29 de Bakker PI, Yelensky R, Pe'er I, Gabriel SB, Daly MJ, Altshuler D: Efficiency and power in genetic association studies. Nat Genet 2005; 37: 1217-1223.

30 Mooy JM, Grootenhuis PA, de VH et al: Prevalence and determinants of glucose intolerance in a Dutch caucasian population. The Hoorn Study. Diabetes Care 1995; 18: $1270-1273$.

31 World Health Organization: Definition, Diagnosis and Classification of Diabetes Mellitus, Report of a WHO Consultation, Part 1: Diagnosis and Classification of Diabetes Mellitus. WHO/NCD/NCS/ 99.2: Geneva, in 1999.

32 van 't Riet E, Rijkelijkhuizen JM, Nijpels G, Dekker JM: Limited agreement between HbA1c and glucose in the general Dutch population: the New Hoorn Study. Diabetologia 2008; 51 (Suppl 1): S164, (abstract).

33 van Tilburg JH, Sandkuijl LA, Strengman E et al: A genome-wide scan in type 2 diabetes mellitus provides independent replication of a susceptibility locus on 18p11 and suggests the existence of novel Loci on 2q12 and 19q13. J Clin Endocrinol Metab 2003; 88: $2223-2230$

34 Monsuur AJ, de Bakker PI, Alizadeh BZ et al: Myosin IXB variant increases the risk of celiac disease and points toward a primary intestinal barrier defect. Nat Genet 2005; 37: 1341-1344.

35 Hofman A, Grobbee DE, de Jong PT, van den Ouweland FA: Determinants of disease and disability in the elderly: the Rotterdam Elderly Study. Eur J Epidemiol 1991; 7: 403-422.

36 Drivsholm T, Ibsen H, Schroll M, Davidsen M, Borch-Johnsen K: Increasing prevalence of diabetes mellitus and impaired glucose tolerance among 60-year-old Danes. Diabet Med 2001; 18: 126-132.

37 Gauderman WJ: Sample size requirements for association studies of gene-gene interaction. Am J Epidemiol 2002; 155: $478-484$.

38 The Diabetes Genetics Initiative of the Broad Institute of MIT and Harvard, Lund University and Novartis Institutes for BioMedical Research. et al: Genome-wide association analysis identifies loci for type 2 diabetes and triglyceride levels. Science 2007; 316: $1331-1336$

39 Zeggini E, Weedon $\mathrm{MN}$, Lindgren $\mathrm{CM}$ et al: Replication of genome-wide association signals in UK samples reveals risk loci for type 2 diabetes. Science 2007; 316: 1336-1341.

40 Zeggini E, Scott LJ, Saxena R et al: Meta-analysis of genome-wide association data and large-scale replication identifies additional susceptibility loci for type 2 diabetes. Nat Genet 2008; 40: 638-645.

41 McCarthy MI, Abecasis GR, Cardon LR et al: Genome-wide association studies for complex traits: consensus, uncertainty and challenges. Nat Rev Genet 2008; 9: 356-369.

42 Sladek R, Rocheleau G, Rung J et al: A genome-wide association study identifies novel risk loci for type 2 diabetes. Nature 2007; 445: 881-885.

43 Scott LJ, Mohlke KL, Bonnycastle LL et al: A genome-wide association study of type 2 diabetes in Finns detects multiple susceptibility variants. Science 2007; 316: 1341-1345.

$44 \mathrm{Ek} \mathrm{J}$, Andersen G, Urhammer SA et al: Mutation analysis of peroxisome proliferator-activated receptor-gamma coactivator-1 
(PGC-1) and relationships of identified amino acid polymorphisms to Type II diabetes mellitus. Diabetologia 2001; 44: $2220-2226$.

45 Krempler F, Esterbauer $\mathrm{H}$, Weitgasser $\mathrm{R}$ et al: A functional polymorphism in the promoter of UCP2 enhances obesity risk but reduces type 2 diabetes risk in obese middle-aged humans. Diabetes 2002; 51: 3331-3335.
46 Barroso I, Luan J, Sandhu MS et al: Meta-analysis of the Gly482Ser variant in PPARGC1A in type 2 diabetes and related phenotypes. Diabetologia 2006; 49: 501-505.

47 Gable DR, Stephens JW, Cooper JA, Miller GJ, Humphries SE: Variation in the UCP2-UCP3 gene cluster predicts the development of type 2 diabetes in healthy middle-aged men. Diabetes 2006; 55: 1504-1511.

Supplementary Information accompanies the paper on European Journal of Human Genetics website (http://www.nature.com/ejhg) 